\title{
Cardiovascular Diseases
}

REVIEW ARTICLE

\section{Clinical Significance and Applications of D-Ribose in Cardiovascular Disease}

\author{
Melike Bayram ${ }^{1}$, David Perkowski², JA St Cyr ${ }^{3}$ and William T Abraham ${ }^{1 *}$ \\ ${ }^{1}$ Cardiovascular Medicine, Ohio State University, USA \\ ${ }^{2}$ Saddleback Memorial Care Center, USA \\ 3Jacqmar, Inc., Minneapolis, USA
}

*Corresponding author: William T Abraham, Cardiovascular Medicine, Ohio State University, 473 W $12^{\text {th }}$, Ave Suite 200, Columbus, OH 43210, USA, Tel: 614-293-8962; 248-798-6485, Fax: 614-293-5614

\begin{abstract}
Cardiovascular disease is the leading cause of deaths worldwide. Myocardial ischemia, a common etiological factor in cardiovascular disease, lowers cellular high-energy phosphate levels, i.e. adenosine triphosphate (ATP), with an accompanying impairment in myocardial function. Furthermore, scientists have argued for the importance of ATP levels in supporting both systolic and diastolic function in heart failure. The magnitude of ischemic heart disease and congestive heart failure is generally well appreciated; however, the scope of myocardial dysfunction may be well underestimated. Clinical investigators have found that approximately half of heart failure patients have preserved ejection fractions with isolated diastolic dysfunction. By improving this myocardial cellular energy deficiency and regenerating myocardial cellular energy levels could offer a potential solution to this problem. D-Ribose (DR), a naturally occurring pentose carbohydrate, has shown promise in pre-clinical and subsequent clinical investigations by regenerating myocardial ATP levels with improving diastolic dysfunction following myocardial ischemia. This review summarizes the uses of DR in pre-clinical studies and clinical applications, both as a diagnostic adjunct and a potential therapeutic agent in cardiovascular diseases.
\end{abstract}

\section{Keywords}

D-ribose, Diastolic dysfunction, Ischemia, Systolic dysfunction, Heart failure

\section{Background}

Cardiovascular disease is the leading cause of deaths worldwide for both males and females. Myocardial ischemia, a common etiological factor in cardiovascular diseases, lowers cellular high-energy phosphate levels, i.e. adenosine triphosphate (ATP), with an accompanying impairment in function. Scientists have argued that the failing heart is energy starved [1,2]. Ingwall and Weiss have argued for the importance of ATP in supporting both systolic and diastolic function in heart failure [1]. While the magnitude of ischemic heart disease and congestive heart failure (CHF) is generally well appreciated, the scope of myocardial dysfunction may be well underestimated. Redfield, et al. reported that systolic and diastolic dysfunction can be present without heart failure symptoms [3]. Approximately half of heart failure patients have preserved ejection fractions with isolated diastolic dysfunction present in more than $40 \%$ of these individuals [4]. Assuming that a myocardial cellular energy deficiency may be present, efforts to regenerate energy levels may offer a needed solution. Myocardial metabolism has been investigated for decades [5]. Supplementation and catabolic enzymatic inhibitors were initially tried to address this metabolic challenge. Decades of research investigating energy substrate supplementation to replenish this myocardial energy deficiency produced mixed findings; however, D-Ribose (DR), a naturally occurring pentose carbohydrate, has shown promise in pre-clinical investigations by regenerating ATP levels and improving diastolic dysfunction following myocardial ischemia. Subsequent clinical applications have supported and expanded these pre-clinical benefits of DR; however, these clinical studies have involved small numbers of patients. 


\section{Reconstituting the ATP Supply}

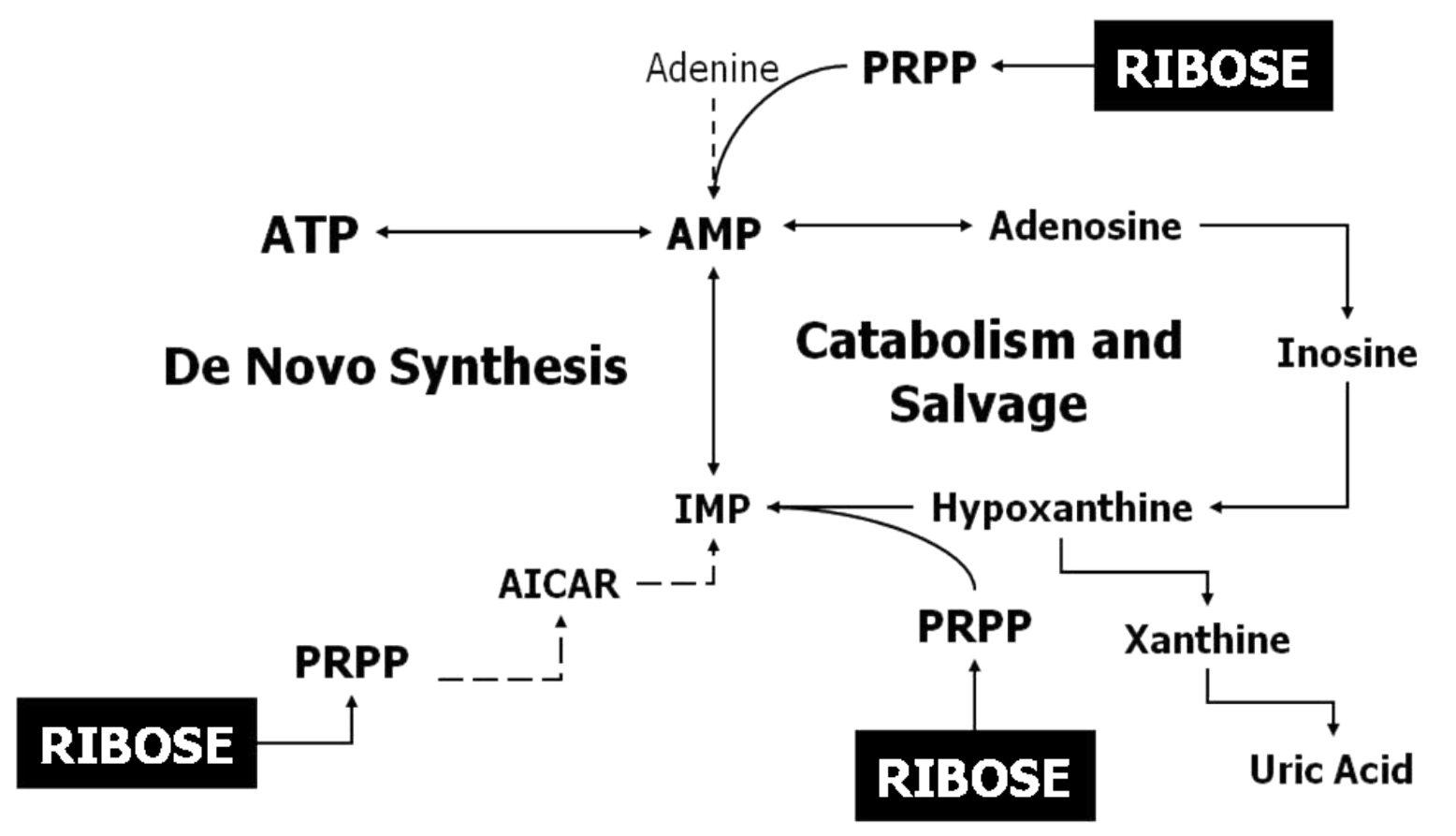

$----\rightarrow$ Denotes intermediate steps

Figure 1: Reconstituting the ATP Supply. Adapted from Ward, et al. [25].

This review summarizes the uses of DR in these pre-clinical studies and clinical applications, both as a diagnostic adjunct and a potential therapeutic agent in cardiovascular diseases.

\section{Myocardial Adenine Nucleotides}

Adenosine triphosphate is an important energy molecule for the maintenance of a cells integrity and function. Myocardial ischemia lowers ATP levels, resulting in reduced function, altered cellular homeostasis, and if severe, decreased myocardial viability $[6,7]$. This depletion in myocardial ATP levels following ischemia persists for hours to days due to slow adenine nucleotide synthesis [8,9]. The conversion of DR in the pentose phosphate pathway (PPP) can aid in producing high-energy phosphate compounds. Supplemental DR bypasses the known rate limiting enzymatic step, glucose-6-phosphate dehydrogenase, in the PPP, stimulating purine nucleotide metabolism, the synthesis of ATP increasing the adenine nucleotide pool (Figure 1) $[10,11]$. Besides the synthesis of ATP, ribose is also important in cellular processes, such as phosphorylated derivatives of ribose can form compounds that aid in cellular metabolism. Further, cyclic adenosine monophosphate, derived from ATP, can act as a messenger for specific cellular signal transduction.

The recovery of ATP levels depends on the availability of the precursor phosphoribosyl-pyrophosphate (PRPP), formed after the initial phosphorylation of DR to DR 5-phosphate.

\section{D-Ribose-a Safe Substrate for Cellular Energy}

D-Ribose, a key component of riboflavin, deoxyribonucleic acid, ribonucleic acid, and ATP [12-14], is rapidly absorbed in the gastrointestinal tract. Between $88-100 \%$ of oral DR is absorbed from the small intestine with minimal first-pass hepatic metabolism. At very high DR doses, only a small amount of DR appears unchanged in the urine [15]. D-Ribose has been found to be safe with few adverse side effects either orally or at 5 hour infusion rates as high as $222 \mathrm{mg} / \mathrm{kg} / \mathrm{hr}$ [16]. Pre-clinical, sub-chronic toxicological, embryotoxicity and teratogenicity assessments have substantiated the safety of DR, including amounts as high as $20 \%$ of the diet $[17,18]$. D-ribose can cause a transient lowering of serum glucose levels when administered either orally or parentally [19]. This paradoxical hypoglycemia occurs presumably by competing with glycogen formation and glycogen breakdown in the liver, along with an acute, un-sustained, spike in insulin secretion. Further, oral doses of DR, exceeding $200 \mathrm{mg} / \mathrm{kg} / \mathrm{hr}$ have the potential to produce mild gastrointestinal symptoms, such as loose stools and rarely, diarrhea.

Advanced glycation end products (AGEs), formed when a reducing carbohydrate reacts with a protein 
or lipid, contribute to an increase state of oxidant stress and inflammation and have been postulated to potentially play a role in chronic disease states, such as atherosclerosis, diabetes mellitus, and chronic renal failure [20]. Pre-clinical animal studies have reported that DR has the potential to produce appreciable levels of AGEs [21,22]. However, quantitative measured levels of AGEs when taking DR and its direct effect on chronic diseases in humans have yet to be thoroughly investigated.

\section{Pre-Clinical Studies}

\section{ATP recovery following ischemia}

Numerous studies have reported that myocardial ATP levels following ischemia do not recover promptly with reperfusion with complete recovery taking hours to days. Kloner, et al. reported that the recovery of ATP levels following 15 minutes of coronary occlusion in otherwise normal, healthy canine hearts was only $75 \%$ complete after three days with complete return requiring one week [23]. Due to this delay in the recovery of ATP, many researchers directed their attention to a metabolic approach to aid in regenerating high energy phosphates. The availability of adequate amounts of ribose in our diets is not appreciable and the cell relies on its synthesis within the cells, which is slow and rate limited due to enzymatic steps. Therefore, supplementation of ribose appeared to be the logical choice in regenerating ATP and to try to satisfy the demand/supply ratio within each cell during and following stressful situations. Zimmer, et al. observed that a continuous infusion of DR following a 15-min period of myocardial ischemia led to a restoration in the cardiac ATP pool within 12 hours in comparison to 72 hours without DR supplementation [24]. Further, Pasque, et al. investigated the acute role of DR in the isolated working rat heart model [10]. Hearts subjected to 15 minutes of ischemia, followed by 15 minutes of reperfusion showed an increase in functional recovery with improved ATP levels post-ischemia with supplemental DR [10].

Ward, et al. reported that myocardial precursor availability is an important limiting factor in ATP recovery, and recovery time following existing or various states of ischemia and also potentially during states of hypoxemia. The permeability of the cellular membrane during these ischemic or hypoxic states allows catabolic products of ATP to be lost from the cell; and therefore, these precursors are not available to aid in regenerating ATP, creating a mismatch in the supply and demand ratio within the cell. Researchers have demonstrated that the recovery time for ATP can be greatly shortened with precursor supplementation [25]. Pre-clinical studies have reported the benefits of DR in enhancing myocardial ATP levels and function following global and regional ischemia [10,11,24,26]. Moderate myocardial global ischemia in a chronic canine model reflected a reduction in ATP levels by $50 \%$. Kriett, et al. showed that with this depletion of ATP levels following myocardial global ischemia, there is an associated state of left ventricular diastolic dysfunction [27]. Pouleur argued that ATP is required for the interaction of calcium with the sarcoplasmic reticulum in muscle cells. Lower cellular levels of ATP may allow calcium to remain fixed to troponin, resulting in a myocardial diastolic dysfunctional state [28].

Beneficial long-term effects in ATP recovery following global myocardial ischemia in a chronic canine model have been reported with adenine and DR supplementation. More than an eightfold increase in de novo ATP synthesis, unlike placebo-controls, was reported with DR ( $2.8 \pm 0.59$ vs. $0.34 \pm 0.06 \mathrm{nmoles} / \mathrm{mg} /$ day, respectively). The return in myocardial ATP levels averaged $9.9 \pm 1.4$ days in controls and only $1.2 \pm 0.2$ days with adenine and DR $[25,29]$. Schneider, et al. reported that besides the improved return in myocardial ATP levels following global ischemia in a chronic canine model, there was also an improvement in diastolic dysfunction with improved left ventricular compliance [30]. D-ribose alone or in combination with adenine significantly enhanced the recovery of ATP levels along with an improvement in diastolic compliance by 24 hours with the recovery of systolic function by four hours. Likewise, Tveter, et al. found myocardial energy and left ventricular functional benefits following moderate ischemia in a chronic canine model with DR alone, when compared to controls (normal saline) [31].

\section{Effects of D-ribose following myocardial infarction}

Stress following a myocardial infarction can cause increasing myocardial dysfunction, chronic cardiac failure and a potential state of apoptosis. The remote myocardium from the infarction site accepts an increased workload and this extra stress can further deplete myocardial energy levels, eventually leading to apoptosis with alterations in myocardial architecture and remodeling. Zimmer, et al. found a progressive decline in left ventricular systolic pressure (LVSP), a decline in left ventricular $\mathrm{dp} / \mathrm{dt}_{\text {max }^{\prime}}$ a compromised rate-pressure product (RPP), elevation in left ventricular end diastolic pressure (LVEDP), and depressed cardiac outputs and stroke volume index following myocardial infarction [11]. Supplemental DR produced an improvement in these hemodynamic parameters and stimulated adenine nucleotide biosynthesis without a further fall in ATP levels in the non-ischemic myocardium within four days after coronary artery ligation [11].

Befera, et al. assessed the potential role of DR in this remote myocardium following acute infarction [32]. They found a significant reduction in left ventricular dysfunction in this remote myocardium following acute infarction. Left ventricular function improved in these remote areas with DR with increased contractility and myocardial wall thickness increase and less ventricular dilation (Table 1). These findings reflect the 
Table 1: Echo Indices in Rats Following Acute MI.

\begin{tabular}{|c|c|c|c|}
\hline Echo Indices & Treatment & Pre-MI & 4 weeks after $\mathrm{Ml}$ \\
\hline \multicolumn{4}{|c|}{ LV diastolic diameter (cm) } \\
\hline & Ribose & $0.64 \pm 0.04$ & $0.76 \pm 0.07^{\star}$ \\
\hline & Control & $0.68 \pm 0.03$ & $0.94 \pm 0.07$ \\
\hline \multicolumn{4}{|c|}{ LV systolic diameter (cm) } \\
\hline & Ribose & $0.39 \pm 0.06$ & $0.64 \pm 0.09^{*}$ \\
\hline & Control & $0.40 \pm 0.03$ & $0.95 \pm 0.07$ \\
\hline \multicolumn{4}{|c|}{ Septal diastolic thickness (cm) } \\
\hline & Ribose & $0.13 \pm 0.02$ & $0.12 \pm 0.02$ \\
\hline & Control & $0.12 \pm 0.01$ & $0.10 \pm 0.01$ \\
\hline \multicolumn{4}{|c|}{ Posterior wall diastolic thickness (cm) } \\
\hline & Ribose & $0.13 \pm 0.02$ & $0.13 \pm 0.03^{*}$ \\
\hline & Control & $0313 \pm 0.03$ & $0.10 \pm 0.01$ \\
\hline \multicolumn{4}{|c|}{ Ejection fraction $(E F)(\mathrm{cm})$} \\
\hline & Ribose & $76.4 \pm 5.9$ & $49.1 \pm 9.4^{*}$ \\
\hline & Control & $76.3 \pm 2.3$ & $31.2 \pm 4.8$ \\
\hline \multicolumn{4}{|c|}{ Shortening fraction (SF) \% } \\
\hline & Ribose & $38.6 \pm 5.3$ & $19.6 \pm 6.1^{*}$ \\
\hline & Control & $38.9 \pm 2.1$ & $10.7 \pm 1.1$ \\
\hline \multicolumn{4}{|l|}{ Note: } \\
\hline${ }^{*} p<0.05$ vs. $c 0$ & & & \\
\hline
\end{tabular}

Adapted from Befera, et al. [32].

potential benefit of DR in preventing or delaying the development of ventricular dysfunction, commonly seen post-infarction and that a potential mechanism in heart failure may reside in decreased myocardial energy levels and not in a breakdown of the heart's contractile apparatus.

\section{Clinical Studies}

\section{Effects of D-ribose on hibernating myocardium}

Pre-clinical benefits have also been observed clinically in cardiovascular medicine, both in the diagnostic and therapeutic arenas. Hibernating myocardium, reflective of chronic ischemia, represents a persistent state of depressed ventricular function associated with decreased blood flow. This chronic myocardial ischemic state is likely associated with low levels of myocardial ATP. Theoretically, the estimation of the extent of myocardial hibernation could aid in management strategies for revascularization. Clinicians have repeatedly stressed that delays in revascularization of hibernating myocardial regions may lead to irreversible cell death with potential adverse outcomes $[33,34]$. Allman, et al. reported a significant relationship between myocardial viability and improved survival after surgical revascularization in patients with left ventricular dysfunction [35].

Various methods are used to assess myocardial hibernation, such as thallium-20l scans, dobutamine stress echocardiography, positron emission tomography, and magnetic resonance imagery. Several studies have reported the benefits of DR in identifying hibernating myocardium. Perlmutter, et al. reported in patients with hibernating myocardium undergoing thalium-201 stress testing that DR identified more reversible defects compared to a control [36]. They concluded that DR probably facilitates 201-thallium redistribution, which is energy dependent, in patients with coronary artery disease (CAD); thereby, enhancing the sensitivity of identifying ischemic myocardium. Hegewald, et al., using SPECT thallium imaging, also found that DR increased the detection of thallium redistribution and the identification of viable myocardium in patients with CAD [37]. These observations using nuclear scan imaging technologies were initially demonstrated in animal studies [38].

More recently, the use of dobutamine stress echocardiography (DSE) in identifying hibernating myocardium also found the benefits of DR. Sawada, et al. reported in 25 patients with ischemic cardiomyopathy that DR improved wall motion identification in dysfunctional segments compared to a placebo group $(63 \%$ versus $45 \%$, respectively; $p=0.02$ ) [39]. At increasing concentrations of dobutamine during DSE, DR reduced the myocardial stress-induced ischemia. Eleven of these study patients subsequently underwent coronary artery bypass grafting and the predicted functional recovery in the DR group was $61 \%$ compared to $46 \%$ in the placebo patients. While not reaching statistical significance, likely due to the small sample size, these results demonstrate a positive encouraging trend. The authors concluded that DR increased the frequency of response to DSE in hibernating myocardial segments and appeared to predict an improvement in overall systolic function in the DR group following revascularization. These observations could have important therapeutic implications. If dysfunctional myocardial segments are more functional with $D R$, the chronic administration of DR could 

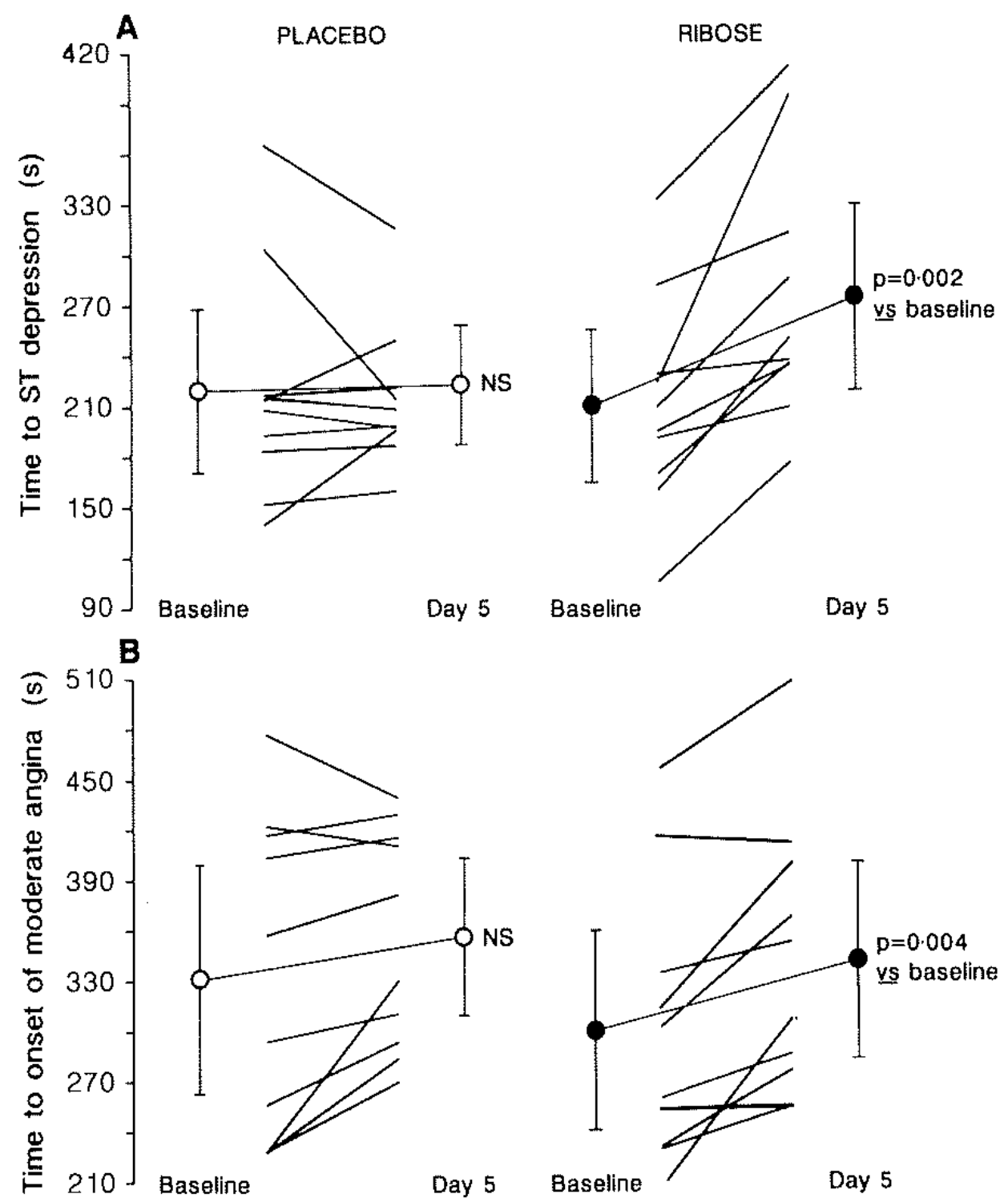

\section{Treadmill walking times until ST-segment depression ( $A$ ) and onset of moderate angina (B).}

Baseline $=$ average of results on days 1 and 2 for each patient; day $5=$ after 3 days treatment. Mean and $95 \%$ confidence intervals given for each group.

Figure 2: Treadmill Walking Times in Patients with Stable CAD. Adapted from Pliml, et al. [40].

conceivably produce a sustained improvement in ventricular function in ischemic myocardium. Moreover, this improved ventricular functional response seen with short-term dobutamine with DR might be exploited in the treatment of decompensated heart failure patients requiring inotropic therapy by potentially protecting the myocardium from excessive depletion of high-energy phosphates. This increases opportunities for new therapies, as well as potentially decreasing the harmful effects of these or similar agents.

\section{Effects of D-ribose in CAD and CHF patients}

Pliml, et al. disclosed that ischemia creates a significant, clinically relevant impairment in ATP levels with no current therapeutic approaches to protect or restore these deficient myocardial energy levels during and following ischemia [40]. Pliml, et al. reported in patients with stable severe coronary artery disease that DR supplementation produced a clinical benefit by significantly increasing treadmill exercise time before the onset of angina and ischemic electrocardiographic changes (Figure 2).

Myocardial ischemia is a major underlying etiological factor in $\mathrm{CHF}$ and recent publications have reported that the failing heart is "energy starved", i.e. deficient of high energy compounds [1,2]. D-ribose has been shown to regenerate low myocardial ATP levels with an accompanying improvement in ventricular function following ischemia. These benefits have also been observed in CHF patients. Commonly, patients with $\mathrm{CHF}$ 
complain of a difficulty in breathing along with constant fatigue. Furthermore, with progression of their failure, patients experience a decrease in ventilatory efficiency (VE), one current term reflecting the efficiency of the cardiopulmonary system. Carter, et al. reported in studying New York Heart Association (NYHA) class II-III CHF patients with left ventricular dysfunction that oral DR significantly maintained $\mathrm{VO}_{2 \max }$ and improved $\mathrm{VE}$ at both anaerobic threshold (AT) and their respiratory compensation point [41]. In addition to these findings, there were positive trends observed in both quality of life and measured B-type Natriuretic Peptide (BNP) levels (Table 2). Likewise, Vijay, et al. also found benefits with DR in VE in NYHA class II-IV CHF patients with a significant improvement in VE in class III and IV patients and a positive beneficial trend in class II patients (Table 3) [42].

Spectrums of pure diastolic, diastolic and systolic, or pure systolic dysfunction occur in patients with CHF. Again, DR has shown to offer benefits in patients with impaired diastolic function. Omran, et al. reported in NYHA class II-III CHF patients that supplemental

Table 2: Ventilatory Changes in Class II \& III CHF Patients.

\begin{tabular}{|l|l|l|l|}
\hline & $\mathrm{VO}_{2} \max$ & $\mathrm{VE} / \mathrm{VCO}_{2} @ \mathrm{AT}$ & $\mathrm{RCP}$ \\
\hline $\mathrm{DR}$ & $-1.4 \pm 8.5$ & $4.9 \pm-6.4$ & $5.8 \pm 9.8$ \\
\hline $\mathrm{PL}$ & $-7.8 \pm 7.3$ & $-6.9 \pm 9.1$ & $-1.4 \pm 8.5$ \\
\hline
\end{tabular}

Note:

RCP $=$ Respiratory compensation point

$\mathrm{VE} / \mathrm{VCO}_{2}=$ Ventilatory efficiency

$\mathrm{VO}_{2} \max =$ Maximal oxygen consumption

$\mathrm{PL}=$ Placebo

Adapted from Carter, et al. [41].

Table 3: Ventilatory Efficiency in CHF Patients.

Ventilatory Efficiency

\begin{tabular}{|l|l|l|}
\hline & Pre-DR & Post-DR \\
\hline Class II, $\mathrm{n}=8$ & $29.4 \pm 5.0$ & $28.8 \pm 4.9$ \\
\hline Class III, $\mathrm{n}=9$ & $43.9 \pm 4.8$ & $38.5 \pm 3.9^{*}$ \\
\hline Class IV, $\mathrm{n}=6$ & $60.4 \pm 5.8$ & $46.1 \pm 6.8^{* *}$ \\
\hline
\end{tabular}

Note:

* $\mathrm{p}<0.05$

** $p<0.005$

Adapted from Vijay, et al. [42]. oral DR improved diastolic dysfunction, quality of life, and physical function assessment. A significant improvement in left atrial contribution with smaller left atrial dimensions, enhanced left atrial velocity time interval, and shortened $\mathrm{E}$ wave deceleration times was found with supplemental DR (Table 4) [43]. These benefits found with DR appear to initiate further studies directed at focusing on an unmet treatment for diastolic dysfunction in patients afflicted with ischemic CHF. More recently Bayram, et al. published an additional study demonstrating the benefits on D-ribose in heart failure patients with preserved ejection fraction and diastolic dysfunction. They found in NYHA class II-IV patients with normal left ventricular systolic function and echocardiographic evidence of diastolic dysfunction that supplementation of DR ( $5 \mathrm{gms} /$ dose) for 6 weeks revealed an improvement in their tissue Doppler velocity values ( $\left.E^{\prime}\right)$ in $64 \%$ of the patients and some patients $(45 \%)$ showed in improvement in their early diastolic filling velocity to early annulus relaxation velocity ratio $\left(E / E^{\prime}\right)$. In addition, $36 \%$ of the study patients had an improvement in their maximum predicted $\mathrm{VO}_{2}$ measurements [44].

\section{Effects of D-ribose in cardiovascular surgical pro- cedures}

D-Ribose has also been investigated for its potential benefits for cardiovascular surgical patients. Diastolic dysfunction can be a problem following surgery. Wyatt, et al. reported that an asanguineous, cardioplegic solution containing adenosine, hypoxanthine, and DR maintained myocardial ATP content during ischemia and with reperfusion, enhancing functional recovery [45]. Vance, et al. investigated the potential role of DR in elective aortic valve replacement with or without associated coronary arterial grafting. Post-operatively, while only $20 \%$ of the patients receiving DR demonstrated a decline in ejection fraction, $80 \%$ of patients in the placebo group demonstrated this decline. They concluded that DR appeared to preserve left ventricular function peri-operatively in patients undergoing aortic valve surgery alone or in combination with coronary artery bypass [46]. These post-surgical benefits of DR have also been observed in patients undergoing coronary arterial revascularization, including patients presenting with

Table 4: Quality of Life \& Physical Function in Class II \& III CHF Patients.

Quality of life, physical function, functional capacity data $(n=15)$

\begin{tabular}{|l|l|l|l|l|}
\hline & Dextrose & \multicolumn{2}{l|}{ Ribose } \\
\hline & Pre & Post & Pre & Post \\
\hline Quality of life & $420 \pm 144$ & $463 \pm 160$ & $417 \pm 118$ & $467 \pm 128^{*}$ \\
\hline Physical function & $52 \pm 20$ & $56 \pm 24$ & $48 \pm 23$ & $54 \pm 21^{* *}$ \\
\hline Funct cap (peak-attained watts) & $102 \pm 19$ & $100 \pm 10$ & $100 \pm 18$ & $103 \pm 15$ \\
\hline Note: & & & & \\
\hline Funct cap = functional capacity & & & & \\
\hline${ }^{*}$ P $<0.01$ & & & & \\
\hline${ }^{*} P<0.02$ & & & & \\
\hline
\end{tabular}

Adapted from Omran, et al. [43]. 
acute myocardial infarction. In 366 patients that underwent off pump coronary artery bypass with the use of a perioperative metabolic protocol, which included $\mathrm{DR}$, demonstrated low mortality and morbidity with a significant early post-operative improvement in cardiac index [47]. Perkowski, et al. also reported that pre-loading patients with oral DR that presented with acute myocardial infarction prior to "off pump" cardiopulmonary bypass revascularization resulted in a significant functional benefit postoperatively with cardiac indices improving $43 \%$ with DR as compared to only a $13 \% \mathrm{im}$ provement in the controls [48].

\section{Summary}

Adenosine triphosphate, an important energy nucleotide, plays a crucial role in many processes for every cell. Many factors are important for the continual production of ATP levels, which can be extremely compromised with stressful conditions, such as ischemia or hypoxia. Researchers have found that following a myocardial ischemic injury, ATP levels can be depressed and adenosine monophosphate (AMP) availability may limit ATP regeneration. Many potential substrates have been investigated to offer a solution to the restoration of ATP during and following stressful conditions. D-ribose has been found to offer this needed benefit. Schematically, via the PPP, DR bypasses key enzymatic steps to enhance the production of ATP levels. Animal studies have demonstrated this increase in myocardial ATP levels following ischemia, as well as demonstrating an improvement in ventricular function with the potential to aid in subsequent chronically needed myocardial remodeling. We acknowledge that subsequent clinical studies investigating the potential beneficial effects of DR have involved a small number of patients in each of these studies with limited statistical power; however, these preliminary studies found encouraging results or benefits with D-ribose supplementation. These studies presented within this paper, in general, imply that DR may potentially offer a natural myocardial energy and functional benefit. We agree that the presented studies in this review are underpowered for placebo-controlled significance; and therefore, placebo-controlled trials, centering on these potential benefits of DR are further needed and essential. Even with the reported benefits from supplementing DR during ischemic or hypoxic conditions, the small patient sampling size produces limitations, which should potentially stimulate researchers to pursue larger cohort studies in the future.

This preliminary review of the available data both pre-clinical and limited clinical investigations gives hope that DR may have desirable effects on many fronts, including an improvement in left ventricular function in the presence of systolic and/or diastolic dysfunction; help salvage viable myocardium around the time of myocardial infarction; attenuate pathological ventricular remodeling; increase exercise tolerance in CHF patients as well as in CAD patients with chronic angina; preserve and potentially improve left ventricular function around the time of surgery; and aid in assessing myocardial viability.

\section{Conclusion}

Ribose, a natural occurring pentose monosaccharide, plays a crucial role in the structure of nucleic acids, our cellular energy cellular metabolism and cellular processes. The continual production in ATP molecules is crucial to maintain cellular integrity and function. Under stress this energy milieu can be altered, in which supply does not meet demand; and therefore, this decreased state of available ATP molecules can affect cellular function, membrane integrity and intracellular processes. Further, states or degrees of ischemia and hypoxemia can alter the cell's membrane to be more permeable, resulting in the loss of the catabolic ATP substances, which limits the potential to regenerate the lower levels of ATP due to the loss of these necessary precursors. Researchers have investigated various precursor substrates, such as adenosine, adenine, inosine and creatine, to aid in this recovery process in ATP levels. However, DR has shown beneficial effects in regenerating ATP levels, unlike other precursors, both in pre-clinical animal and clinical studies. These studies have repeatedly demonstrated the safety of $D R$, and supplementation of DR has shown to replenish myocardial ATP levels with an improvement in diastolic function following ischemia, including patients with CHF. From these preliminary investigations, DR as a natural metabolic supplement, appears to be useful as a diagnostic adjunct in unmasking hibernating myocardium and, more importantly, as a therapeutic "energy modulator" in the treatment of cardiovascular diseases, including surgical intervention.

Therefore, the clinical applications of this natural occurring pentose molecule could have many benefits in patients afflicted with ischemic cardiovascular diseases. Due to the nature of this disease and knowing that adequate levels of energy molecules are paramount in maintaining cellular integrity and function, D-ribose could offer a means to satisfy the supply and demand mismatch that is commonly found during and following ischemia. D-ribose could potentially provide a solution to this ongoing problem in patients afflicted with ischemic cardiovascular disease.

\section{References}

1. Ingwall JS, Weiss RG (2004) Is the failing heart energy starved? On using chemical energy to support cardiac function. Cir Res 95: 135-145.

2. Neubauer $S$ (2007) The failing heart-an engine out of fuel. N Engl J Med 356: 1140-1151.

3. Redfield MM, Jacobson SJ, Burnett JC, Mahoney DW, Bailey KR, et al. (2003) Burden of systolic and diastolic ventricular dysfunction in the community. Appreciating the scope of the heart failure epidemic. JAMA 289: 194-202. 
4. Bursi F, Weston SA, Redfield MM, Jacobsen SJ, Pakhomov S, et al. (2006) Systolic and diastolic heart failure in the community. JAMA 296: 2259-2260.

5. Weiss RG, Maslov M (2004) Normal myocardial metabolism: Fueling cardiac contraction. Adv Stud Med 4: S457-S463.

6. Rahimtools SH (1989) The hibernating myocardium. Am Heart J 117: 211-221.

7. Ross J (1991) Myocardial perfusion-contraction matching Implications for coronary heart disease and hibernation. Circulation 83: 1076-1083.

8. Mahoney JR (1990) Recovery of post ischemic myocardial ATP levels and hexosemonophosphate shunt activity. Med Hypoth 31: 21-23.

9. Ibel H, Zimmer HG (1986) Metabolic recovery following temporary regional myocardial ischemia in the rat. $\mathrm{J} \mathrm{Mol}$ Cell Cardiol 18: 61-65.

10. Pasque MK, Spray TL, Pellom GL, Van Trigt P, Peyton $\mathrm{RB}$, et al. (1982) Ribose-enhanced myocardial recovery following ischemia in the isolated working rat heart. $J$ Thorac Cardiovasc Surg 83: 390-398.

11. Zimmer HG, Martius PA, Marschner G (1989) Myocardial infarction in rats: Effects of metabolic and pharmacologic interventions. Basic Res Cardiol 84: 332-343.

12. Stryer L (1988) Carbohydrates. In: Stryer L, Biochemistry. ( $3^{\text {rd }}$ edn), W H Freeman and Company, New York, 331-337.

13. Anderson DM (2000) Dorland's Illustrated Medical Dictionary. (29th edn), W B Saunders Company, Philadelphia, 1576.

14. Marcus R, Coulston AM (1990) Water-soluble vitamins: The vitamin B complex and ascorbic acid. In: Gilman AG, Rall TW, Nies AS, Taylor P, Goodman and Gilman's The Pharmacological Basis of Therapeutics. ( $8^{\text {th }}$ edn), Pergamon Press, New York, 1530-1536.

15. http://WWW.pdrhealth.com/drug_info/nmdrugprofiles/ nutsupdrugs/dri_0226.shtml.

16. Segal S, Foley J (1958) The metabolism of D-ribose in man. J Clin Invest 37: 719-735.

17. Griffiths JC, Borzelleca JF, St Cyr J (2006) Lack of oral embryotoxicity/teratogenicity with D-ribose in Wistar rats. Food Chem Toxicol 45: 388-395.

18. Griffiths JC, Borzelleca JF, St Cyr J (2006) Sub-chronic (13-week) oral toxicity study with D-ribose in Wistar rats. Food Chem Toxieol 45: 144-152.

19. Gross M, Zollner N (1991) Serum levels of glucose, insulin, and C-peptide during long-term D-ribose administration in man. Klinische Wochenschrift 69: 31-36.

20. Kalousova M, Zima T, Tesar V, Dusilova-Sulkova S, Skrha $\mathrm{J}$ (2005) Advanced glycoxisation end products in chronic diseases-clinical chemistry and genetic background. Mutat Res 579: 37-46.

21. Han C, Lu Y, Wei Y, Liu Y, He R (2011) D-ribose induces cellular protein glycation and impairs mouse spatial cognition. PLoS One 6: E24623.

22. Wei Y, Han CS, Zhou J, Liu Y, Chen L, et al. (2012) D-ribose in glycation and protein aggregation. Biochim Biophys Acta 1820: 488-494.

23. Kloner RA, DeBoer LW, Darsee JR, Ingwall JS, Braunwald $E$ (1981) Recovery from prolonged abnormalities of canine myocardium salvaged from ischemic necrosis by coronary reperfusion. Proc Natl Acad Sci USA 78: 7152-7156.
24. Zimmer HG, Ibel H (1984) Ribose accelerates the repletion of the ATP pool during recovery from reversible ischemia of the rat myocardium. J Mol Cell Cardiol 16: 863-866.

25. Ward HB, St Cyr JA, Cogordan JA, Alyono D, Bianco RW, et al. (1984) Recovery of adenine nucleotide levels after global myocardial ischemia in dogs. Surgery 96: 248-255.

26. Clay MA, Stewart-Richardson P, Tasset DM, Williams JF (1988) Chronic alcoholic cardiomyopathy, Protection of the isolated ischaemic working heart by ribose. Biochem Intl 17: $791-800$

27. Kriett JM, Ward HB, Bianco RW, Einzig S, Alyono D, et al. (1983) Recovery of adenine nucleotides and cardiac function following ischemia. Circ 68: 389 .

28. Pauly DF, Pepine CJ (2000) D-ribose as a supplement for cardiac energy metabolism. Cardiovasc Pharmacol 5: 249258.

29. St Cyr J, Bianco RW, Schneider JR, Mahoney JR, Tveter $\mathrm{K}$, et al. (1989) Enhanced high energy phosphate recovery with ribose infusion after global myocardial ischemia in a canine model. J Surg Res 46: 157-162.

30. Schneider JR, St Cyr JA, Mahoney JR, Bianco RW, Ring WS, et al. (1985) Recovery of ATP and return of function after global ischemia. Circ 72: 298.

31. Tveter K, St Cyr JA, Schneider J, Bianco R, Foker J (1988) Enhanced recovery of diastolic function after global myocardial ischemia in the intact animal. Pediatr Res 23: 226.

32. Befera N, Rivard A, Gatlin D, Zhang J, Foker JE (2007) Ribose treatment helps preserve function of the remote Myocardium after Myocardial infarction. J Surg Res 137: 156.

33. Beanlands RS, Hendry PJ, Masters RG, DeKemp RA, Woodend K, et al. (1998) Delay in revascularization is associated with increased mortality rate in patients with severe left ventricular dysfunction and viable myocardium on fluorine 18-fluorodeoxyglucose positron emission tomography imaging. Circ 9: 51-56.

34. Carluccio E, Biagioli P, Alunni G, Murrone A, Giombolini C, et al. (2006) Patients with hibernating myocardium show altered left ventricular volumes and shape, which revert after revascularization: Evidence that dyssynergy might directly induce cardiac remodeling. J Am Coll Cardiol 47: 969-977.

35. Allman KC, Shaw LJ, Hachamovitch R, Udelson JE (2002) Myocardial viability testing and impact of revascularization on prognosis in patients with coronary artery disease and left ventricular dysfunction: a meta-analysis. J Am Coll Cardiol 39: 1151-1158.

36. Perlmutter NS, Wilson RA, Angello DA, Palac RT, Lin J, et al. (1991) Ribose facilitates thallium-201 redistribution in patients with coronary artery disease. J Nucl Med 32: 193-200.

37. Hegewald MG, Palac RT, Angello DA, Perlmutter NS, Wilson RA (1991) Ribose infusion accelerates thallium redistribution with early imaging compared with late 24 hour imaging without ribose. J Am Coll Cardiol 18: 1671-1681.

38. Angello DA, Wilson RA, Gee D (1988) Effect of ribose on thallium-201 myocardial redistribution. J Nucl Med 29: 1943-1950.

39. Sawada SG, Lewis S, Kovacs R, Khouri S, Gradus-Pizlo I, et al. (2009) Evaluation of the anti-ischemic effects of D-ribose during dobutamine stress echocardiography: a pilot study. Cardiovasc Ultrasound 7: 5. 
40. Pliml W, Von Arnim T, Stablein A, Hofmann H, Zimmer HG (1992) Effects of ribose on exercise-induced ischaemia in stable coronary artery disease. Lancet 340: 507-510.

41. Carter O, Mac Carter D, Mannebach S, Biskupiak J, Stoddard G, et al. (2005) D-Ribose improves peak exercise capacity and ventilatory efficiency in heart failure patients. J Am Coll Cardiol 45: 185.

42. Vijay N, MacCarter D, Washam M, Munger M, St. Cyr J (2005) D-Ribose improves ventilatory efficiency in Congestive Heart Failure NYHA Class II-IV patients. Abstract HFSA 11: 95.

43. Omran H, Illien S, MacCarter D, St. Cyr J, Luderitz B (2003) D-Ribose improves diastolic function and quality of life in congestive heart failure patients: A prospective feasibility study. Eur J Heart Failure 5: 615-619.

44. Bayram M, St.Cyr JA, Abraham WT (2015) D-Ribose aids heart failure patients with Preserved ejection fraction and diastolic dysfunction: A pilot study. Ther Adv Cardiovasc Dis 9: $56-65$.

45. Wyatt DA, Ely SW, Lasley RD, Walsh R, Mainwarning R, et al. (1989) Purine-enriched asangiuneous cardioplegia retards adenosine triphosphate degradation during ischemia and improves postischemic ventricular function. $\mathrm{J}$ Thorac Cardiovasc Surg 97: 771-778.

46. Vance RA, Einzig S, Kreisler J, St Cyr J (2000) D-ribose maintains ejection fraction following aortic valve surgery. FASEB J 14: 419

47. Perkowski D, Wagner S, Schneider JR, St Cyr JA (2011) A targeted metabolic protocol with D-ribose for off pump coronary artery bypass procedures: A retrospective analysis. Ther Adv Cardiovasc Dis 5: 185-192.

48. Perkowski D, Wagner S, St Cyr J (2007) D-Ribose and "off' pump coronary artery revascularization aids cardiac indices following acute myocardial infarction. J Heart Dis 5: 92. 